\title{
Medication Error and Interprofessional Communication-Related Factors Contributing to Hospitalisation in Community-Dwelling Older Adults in Australia
}

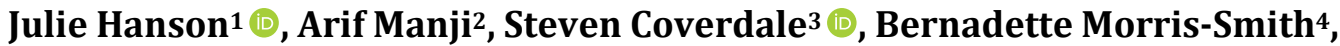 \\ Marianne Wallis ${ }^{1}$ (D) \\ ${ }^{1}$ School of Nursing, Midwifery and Paramedicine, University of the Sunshine Coast, Queensland, Australia \\ ${ }^{2}$ Department of General Medicine, Sunshine Coast Hospital and Health Service, Queensland, Australia \\ ${ }^{3}$ School of Medicine, Sunshine Coast, Griffith University, Queensland, Australia \\ ${ }^{4}$ Sunshine Coast Hospital and Health Service, Queensland, Australia \\ Email: jhanson@usc.edu.au
}

How to cite this paper: Hanson, J., Manji, A., Coverdale, S., Morris-Smith, B. and Wallis, M. (2021) Medication Error and Interprofessional Communication-Related Factors Contributing to Hospitalisation in Community-Dwelling Older Adults in Australia. Open Journal of Nursing, 11, 896-907.

https://doi.org/10.4236/ojn.2021.1110073

Received: August 12, 2021

Accepted: October 25, 2021

Published: October 28, 2021

Copyright $\odot 2021$ by author(s) and Scientific Research Publishing Inc. This work is licensed under the Creative Commons Attribution International License (CC BY 4.0).

http://creativecommons.org/licenses/by/4.0/

(c) (7) Open Access

\begin{abstract}
Background: Population ageing is a worldwide phenomenon. It is common for older adults to develop multiple age-related illnesses and the prevalence of multimorbidity increases substantially with age. Multimorbid adults are frequently treated with several concurrent medications and the regimen may be complex requiring multiple steps in the preparation of a medication prior to its administration. Polypharmacy is a concerning trend and older adults have a $100 \%$ risk of experiencing adverse drug events when taking ten or more medications concurrently. Discharge summaries communicating the number of medications, changes made to medication regimens during hospitalisations and the requirement for ongoing monitoring in the community are often incomplete. The aim of this study was to investigate contributing factors to medication-related hospitalisation, length of stay or readmission in older community-dwelling persons and examine the quality of discharge summaries. Methods: Descriptive and correlational analyses of demographic, clinical, admission, readmission, length of stay and medication variables were examined in Australia in 2016-2018. Discharge summaries were analysed for completeness, timeliness and interprofessional communication. Results: There were 295 participants, mean age 80 years, 55\% were female, taking an average of 11 prescribed medications and with a mean Medication Regimen Complexity Index score of 34. Medication errors that were unrecognised at the time of hospitalisation were present in $19 \%$ of the sample. Factors associated with medication error were older age and a longer median length of stay. Fewer
\end{abstract}


than $52 \%$ of these older patients had detailed discharge summaries. Conclusion: The prevalence of polypharmacy and medication regimen complexity at admission was high. A high proportion of older adults on medical units may have unrecognised medication errors impacting their admission. Medical discharge summaries are inadequately addressing this issue for patients returning to the care of their family physician.

\section{Keywords}

Medication Error, Aged, Hospital Admission, Length of Stay, Discharge Communication

\section{Introduction}

Medication error (ME) is a significant cause of adverse drug events (ADEs) [1]. An ADE can be defined as an undesirable pharmacological effect of a drug when administered at the recommended dose or, an unintentional overdose and ADE risk increases by $10 \%$ with each medication ingested, coming closer to $100 \%$ risk with ten or more medications [2]. Older adults experience more ADEs than younger adults because of age-related physiological and pharmacokinetic changes that reduce that body's ability to deliver drugs to their target organs (distribution), decreased capacity to break down drugs (metabolism) or changes to the way in which the body removes waste products (clearance) [3]. These physiological and pharmacokinetic changes are compounded by increased prevalence of multimorbidity and a concomitant increase in the number of medications prescribed in this population [4]. In the patient safety literature, inappropriate medication use and polypharmacy are reported to be major factors that put older adults at risk for ADEs [5], in addition to decreased social support for this population [6]. The high-risk profile of older adults related to medication administration error results in increased health care utilisation and emergency department presentations [7] [8]. In the international literature, other contributing factors are reported to be advancing age, multimorbidity, dementia, frailty and limited life expectancy [9]. A retrospective study of Department of Veterans' Affairs health records, aimed at identifying avoidable hospitalisations, coincidentally identified that in veterans aged 65 years and over, just over $20 \%$ of admissions were due to potentially preventable medication-related events [10].

One further issue implicated in hospitalisations from medication-related events is the quality of discharge communication. In a pilot study examining the relationship between cognitive impairment and medication self-management errors in older persons discharge home from the hospital, $87 \%$ of patients reported that they were not having any difficulty self-medicating post-discharge, but over half were discovered to have one or more medication discrepancies [11]. Medication discrepancies are defined as inconsistencies between two or more medictaion lists. Discrepancies commonly occur among older patients at discharge and are 
often the result of poorly communicated information or inadequacies in the physicians' discharge summary [12].

The rationale for undertaking this study is that the Sunshine Coast, in South East Queensland (SEQ) has an ageing population that is higher than the national average, comprising $18 \%$ aged 65 years and over compared with $14 \%$ nationally [13]. It is estimated that this will increase to $21.8 \%$ by 2026 , while the proportion of the population aged 85+ is expected to treble [14]. Population ageing in Australia has been increasing steadily since 1970. It is predicted to continue because of the increase in life expectancy and, post-war baby boomers are now entering the older age group (65 years and older). The proportional effect of this is greatest on the two oldest age groups, 80 - 84 years and 85 years and over. These age groups have respectively increased their proportion of the older population by $22 \%$ and $66 \%$ since 1991 , and by $29 \%$ and $14 \%$ since 1971 [13]. Although the increasing ageing population is a national phenomenon, the impact is reported to be felt earlier and more profoundly in coastal communities as the population in these localities continues to grow [14]. These communities are generally older, have more of the very old and have many self-funded retirees who have moved away from family support [14]. This study is significant because no previous data on this subject exists in this region. Arguably, the coastal communities of SEQ have a demographic that provides a view of the future demographic for Australia and highlights the need for immediate medication safety interventions in countries with similar health systems and burgeoning older, multimorbid populations.

\section{Aims}

The study aimed to investigate contributing factors to medication-related hospitalisation, length of stay or readmission in older community-dwelling persons and examine the quality of discharge summaries. The critical variables of interest were the number of medications, regimen complexity and co-morbidities.

\section{Methods}

\subsection{Study Design and Setting}

A retrospective study was used and was based on a chart review. The data were collected from patient medical records after initial presentation to hospital from a community setting. Two regional hospitals in South-East Queensland were used: Hospital A, a tertiary referral hospital with approximately 400 acute and sub-acute beds and Hospital B, a 450 bed tertiary teaching hospital that opened in April 2017. A convenience, sequential sample of patients were included the study following admission to the Medical Assessment and Planning Unit (MAPU).

\subsection{Participants}

Participants eligible for the study included adults, aged 65 years or over, hospitalised between July 2016 and July 2018. All patients admitted to the MAPU during the study period were assessed for inclusion. Many patients are admitted for 
investigation and it is only after assessment that it becomes clear that a medication error was implicated in the reason for admission. Consequently, on admission patients were assessed by the admitting physician who determined which of the study participants had some degree of medication error that contributed to the reason for admission. As a class of medication error, accidental poisoning by drugs was an inclusion criterion if explanatory text was available for review. Participants were excluded if admitted with a diagnosis of suicide attempt since it was not considered to be a class of medication error. The pre-existing data in the medical records of all eligible patients were reviewed by trained nursing personnel and made accessible to the principal investigator once all documents were de-identified. The medical records of all eligible patients were accessed.

\subsection{Sample Size}

$\mathrm{G}^{*}$ power ${ }^{\mathrm{TM}}$ software program was used to calculate sample size [15]. For a linear model including eight variables with small to moderate effect and $95 \%$ power a sample of $n=236$ was required. To allow for the exclusion of patients with incomplete medical records a cohort of 300 patients who were admitted to the designated hospital sites in the defined study period were included, was identified and their records were accessed.

\subsection{Data Collection}

Data of age, gender, reason for admission/diagnosis, co-morbid conditions, medications taken prior to admission, length of stay and time to readmission within 28 days were collected. Discharge summaries completed for the target admission were accessed and textual data abstracted. The names of all medications prescribed during the target admission were collected to assess the quality of the discharge summary using seven criteria that included: whether the summary was complete; completed within 48 hours of discharge; directed to allied health professionals; directed to the General Practitioner (GP-family physician); lists patients' medications; changes made to medications during the hospital stay and identifies any medication monitoring.

If the treating physician had identified that a potential medication error had contributed to admission, the medication error was classified into four distinct groups using the categories developed by the research team. These were:

Group A-Self-administered or carer-administered medication error;

Group B-Incorrectly prescribed medication error;

Group C-Physiological reactions to medication;

Group D-Control (hospital admissions for an event not related to medication).

The Charlson Comorbidity Index (CCI) was calculated from the audited data. $\mathrm{CCI}$ is a diagnosis-based score used to predict 10 -year survival demonstrating that increasing frequency and severity of co-morbidities shortens life expectancy. A weighting is assigned to each of the 17 conditions (in 19 disease categories), 
plus one point for every decade of age over 50 years, to a maximum of 4 points. The sum of the weighted conditions provides a numeric comorbidity score (range: 0 to 33) for each patient [16]. Simply put, a higher score indicates that the patient is older and sicker. The Charlson Comorbidity Index (CCI) possesses face and content validity, good interrater and test-retest reliability, and excellent predictive validity for mortality from co-morbid indices in medical records [17] [18].

The Medication Regimen Complexity Index (MRCI) score was also calculated. MRCI is used to quantify the complexity of each medication regimen. The 65 -item instrument assigns complexity scores to the medication regimen of individual patients based on medication dosage forms, dosing frequencies, and additional administration instructions, such as the specified time of administration, or if food is advised with the medication or not. It is composed of 3 separate sections in order to capture data on dosage forms (section A), dosing frequency (section B) and additional directions (section C). The total score is equal to the sum of the weighted scores of all 3 sections. The minimum index for someone on a medication is 1.5 and there is no established maximum because the number of medications varies from person to person. A higher score indicates a more complex medication regimen. The tool has both convergent (Spearman's rho $=0.9, \mathrm{p}<0.0001)$ and discriminate validity (Spearman's rho $=$ $0.34, \mathrm{p}=0.1$ for age and $\mathrm{p}=0.487$ for gender) and interrater and test-retest reliabilities were greater than or equal to 0.9 for both the total test and the subscale evaluations [19].

\subsection{Ethics Approval}

The study was approved by the Health Service Human Research Ethics Committee (HREC)/16/QPCH/153 and University HREC A16874 and included site-specific approval and a waiver of consent.

\subsection{Data Analysis}

Data were analysed according to the 4 research aims using IBM $^{\mathrm{TM}}$ SPSS Statistics version 24. Continuous variables are presented as mean \pm standard deviation or median (range) and categorical variables as frequency and percentage. Comparisons between groups were assessed using Mann-Whitney tests. The association between nominal variables was performed using the Chi-square test and comparisons of interval data (CCI, MRCI, number of medications).

\section{Results}

\subsection{Sample Characteristics}

Three hundred individuals were eligible for the study and data were available for $98 \%(n=295)$ of these patients. Five participants were excluded from the study due to missing data. Table 1 shows the demographic and clinical characteristics of participants. The mean age was 79.8 years and there were more women (55\%) 
Table 1. Characteristics of participants in the four groups $(n=295)$.

\begin{tabular}{lccccc}
\hline \multicolumn{1}{c}{ Variable } & $\begin{array}{c}\text { Group without } \\
\text { medication error } \\
\mathrm{n}=244\end{array}$ & $\begin{array}{c}\text { Group with } \\
\text { medication error } \\
\mathrm{n}=51\end{array}$ & $\begin{array}{c}\text { Self- or } \\
\text { carer-administered } \\
\text { medication error }\end{array}$ & $\begin{array}{c}\text { Physiological reaction } \\
\text { to medication }\end{array}$ & $\begin{array}{c}\text { Total } \\
\mathrm{n}=295 \\
\text { Age [Mean/SD] }\end{array}$ \\
Female [Frequency/\%] & $131(54 \%)$ & $32(62 \%)$ & $12(60 \%)$ & $20(67 \%)$ & $163(55 \%)$ \\
CCI Score [Mean/SD] & $5.6(2.0)$ & $5.4(2.1)$ & $4.8(2.1)$ & $5.9(2.1)$ & $5.6(2.1)$ \\
MRCI Score [Mean/SD] & $34.8(19.5)$ & $31.8(17.6)$ & $28.4(15.8)$ & $34.2(19.1)$ & $34.3(19.2)$ \\
No. of prescribed medications [Mean/SD] & $11.1(0.1)$ & $10.3(0.5)$ & $8.6(0.4)$ & $11.6(0.5)$ & $11.0(0.0)$ \\
\hline
\end{tabular}

Sample sizes for some of these calculations vary due to missing data.

than men in the sample. The mean co-morbidity score was 5.6 and the mean number of prescribed medications was 11.0. More of the self- or carer-administered medication errors (60\%) and physiological reactions to medications (67\%) occurred in women. All groups had similar CCI scores but the group without medication error had the higher medication complexities. Only one participant presented with a medication error that was due to an incorrectly prescribed medication and this data is not included in Table 1 or Table 3.

Table 2 shows the frequency of comorbid conditions of the sample participants at the time of hospitalisation. Data are organised using thirteen categories and are adapted from the International Classification of Diseases Diagnosis Codes presented in the Charlson Comorbidity Index [16]. Cardiovascular diseases that include hypertension, atrial fibrillation and myocardial infarction were the most frequently reported comorbidities at $71.9 \%$, with joint and connective tissue conditions such as osteoarthritis at $36.6 \%$, followed by hyper- and dyslipidaemia at $33.9 \%$. Gastrointestinal conditions that included gastro-oesophageal reflux disease and diverticulitis occurred in $29.8 \%$ of participants and obesity $2.0 \%$ in this population.

\subsection{Length of Stay and Readmission}

In Table 3 the median length of stay was three days (Interquartile range $(\mathrm{IQR})=$ 5) for patients without medication error and four days $(I Q R=10)$ for those with medication error. This difference was not statistically significant (MWU = 5688.5; $\mathrm{p}=0.33$ ). The longest length of stay was for those admitted with self-administered medication error with a median of 8.5 days $(\mathrm{IQR}=15)$. In total 97 (32\%) of the sample were readmitted within 28 days. A smaller proportion of those whose admission was associated with a medication were re-admitted compared to the group without medication error (26\% versus $34 \%$ ).

\section{Factors associated with length of stay}

No association was found between length of stay and CCI score $(\mathrm{r}=0.007 ; \mathrm{p}=$ 0.914); MRCI score $(r=0.009 ; \mathrm{p}=0.886)$; or number of prescribed medications $(r=-0.24 ; p=0.697)$. There was a slight negative association between age and 
Table 2. Characteristics of participants' comorbidities $(n=295)$.

\begin{tabular}{lc}
\hline & $\mathrm{n}(\%)$ \\
\hline Cardiovascular & $212(71.9)$ \\
Renal & $34(11.5)$ \\
Endocrine & $61(20.7)$ \\
Gastrointestinal & $88(29.8)$ \\
Liver/metabolic & $64(21.7)$ \\
Respiratory/pulmonary & $70(23.7)$ \\
Malignancy & $79(26.8)$ \\
Depression/mental health/anxiety & $42(14.2)$ \\
Dementia/cognitive impairment & $40(13.6)$ \\
Peripheral vascular & $36(12.2)$ \\
Cerebrovascular & $48(16.3)$ \\
Joint and connective tissue & $108(36.6)$ \\
Obesity & $6(2.0)$
\end{tabular}

Table 3. Statistics for readmission rates and LOS.

\begin{tabular}{cccccc}
\hline Variable & $\begin{array}{c}\text { Group without } \\
\text { medication error } \\
\mathrm{n}=244\end{array}$ & $\begin{array}{c}\text { Group with } \\
\text { medication error } \\
\mathrm{n}=51\end{array}$ & $\begin{array}{c}\text { Self-or carer } \\
\text { administered } \\
\text { medication error }\end{array}$ & $\begin{array}{c}\text { Physiological reaction } \\
\text { to medication }\end{array}$ & $\begin{array}{c}\text { Total } \\
\mathrm{n}=295\end{array}$ \\
\hline Readmission [Frequency/\%] & $84(34 \%)$ & $13(26 \%)$ & $5.0(25 \%)$ & $7.0(23 \%)$ & $295(100 \%)$ \\
Length of Stay (Days) [Median/IQR] & $3.0(5.0)$ & $4.0(10.0)$ & $8.5(15.0)$ & $2.0(8.0)$ & $3.0(6.0)$ \\
\hline
\end{tabular}

Length of stay was positively skewed $>1.96$ (non-parametric test median and interquartile range used).

length of stay $(\mathrm{r}=-0.14 ; \mathrm{p}=0.02)$ and, although statistically significant, this association means that only $2 \%$ of the variability in length of stay is explained by the variability in age which appears to be clinically insignificant. A point-biserial correlation was undertaken to determine the relationship between length of stay and gender and there was no correlation $\left(\mathrm{r}_{\mathrm{pb}}=0.021, \mathrm{p}=0.751\right)$.

\section{Readmission}

There were no statistically significant associations between readmission and MRCI score $\left(\mathrm{r}_{\mathrm{pb}}=-0.017 ; \mathrm{p}=0.78\right)$ nor number of medications prescribed $\left(\mathrm{r}_{\mathrm{pb}}=\right.$ $0.020 ; \mathrm{p}=0.75)$. In addition, there were no statistically significant relationships between gender $\left(\chi^{2}(\mathrm{df})=0.42(1) ; \mathrm{p}=0.52\right)$ age $\left(\mathrm{r}_{\mathrm{pb}}=0.091 ; \mathrm{p}=0.12\right)$ and CCI score $\left(\mathrm{r}_{\mathrm{pb}}=0.067 ; \mathrm{p}=0.29\right)$ and readmission.

\subsection{Quality of Discharge Summaries}

Table 4 presents the frequency and percentage results related to the quality of discharge summaries provided for the two groups of patients, those with and without medication error as a factor in their admission. In general, those who had a medication error implicated in their admission had a higher frequency of completed discharge summaries and more information was included about their medication regimen compared to the group without medication error. However, proportions of patients that had detailed summaries that listed medications, 
Table 4. Frequencies and percentages of aspects of discharge summaries.

\begin{tabular}{lccc}
\hline \multicolumn{1}{c}{ Discharge summary information } & $\begin{array}{c}\text { No medication error } \\
(\mathrm{n}=244) \\
{[\text { Frequency } \%]}\end{array}$ & $\begin{array}{c}\text { Medication error } \\
(\mathrm{n}=51) \\
{[\text { Frequency \%] }}\end{array}$ & $\begin{array}{c}\text { Total } \\
(\mathrm{n}=295) \\
{[\text { Frequency \%] }}\end{array}$ \\
\hline Completed & $229(94 \%)$ & $49(96 \%)$ & $278(94 \%)$ \\
Completed within 48 hours of discharge & $197(81 \%)$ & $43(84 \%)$ & $240(81 \%)$ \\
Directed at allied health professional & $2.00(0.8 \%)$ & $3.0(6 \%)$ & $5.00(2 \%)$ \\
Directed to GP & $165(68 \%)$ & $36(71 \%)$ & $201(68 \%)$ \\
Summary lists patient's medications & $121(50 \%)$ & $31(61 \%)$ & $152(52 \%)$ \\
Summary identifies change to medication regimen during recent stay & $111(46 \%)$ & $28(55 \%)$ & $139(47 \%)$ \\
Summary identifies any medication related monitoring & $85.0(35 \%)$ & $21(41 \%)$ & $106(369 \%)$ \\
\hline
\end{tabular}

changes to medications and identified medications requiring monitoring was less than $52 \%$ overall.

\section{Discussion}

This preliminary study was undertaken in a coastal area of Australia with a high population of older adults. It investigated hospitalisations of older adults where a medication error, in the community, was subsequently found to be a contributing factor to hospital admission. In this study, $17 \%(\mathrm{n}=51)$ had a medication error-related reason for their hospitalisation, most occasions of which were not identified prior to hospitalisation. The results revealed a mean age of 80 years and an average co-morbidity (CCI) score of 5.6 revealing an estimated 10-year survival of $2 \%-21 \%$ [16]. Similarly, a retrospective chart audit of 164 patients (aged 50 years and over) in major metropolitan teaching hospital in Australia revealed a mean age of 74 years, high co-morbidity score using the CCI, a median of nine prescribed medications with $20 \%$ of patients from residential care and $37 \%$ living alone [20].

On average, our patients were taking 11.0 prescribed medications and the mean MRCI score was high at 34.3 [19]. Polypharmacy is a concerning trend and these results mirror larger epidemiological studies conducted in other countries with similar health systems, such as Scotland [21]. One significant concern is the $100 \%$ risk of $\mathrm{ADE}$ when patients take ten or more medications concurrently, as seen in this study, and is consistent with earlier research [2].

In our study, we found that there was no statistically significant difference in length of stay between those admitted with and without medication error. The longest length of stay was for those admitted with self-administered medication error. However, the greater proportion of those readmitted within 28 days were patients in the group without medication error $(n=244)$ rather than those with medication error $(n=51)$. The group without medication error was over 4.5 times larger than the group with a medication-related reason for hospitalisation, the mean age was 80 years old and the average $\mathrm{CCI}$ was high at 5.6. These factors 
may explain this study finding since multimorbidity and advancing age are reported to increase healthcare utilisation [9]. In addition, demographic and clinical factors are recognised as significant contributing factors to readmission rates at 30-, 60- or 90-day periods in this population and these timeframes are the most frequently researched [6].

Our study suggests a high rate of availability of discharge summaries within 48 hours ( $83 \%$ in the medication associated group). However, there remains substantial room for improvement in availability of medication lists, documentation of changes to medications and guidance about the monitoring required since the proportions of patients that had detailed summaries was less than 52\% of the study sample. The literature reveals mounting unease about the quality of interprofessional communication between hospital and primary care providers particularly where medication changes have been made during the hospital stay. In one study, $95 \%$ of patients had a discharge summary but for $19 \%$ the summaries were not completed or transmitted to primary care givers within two weeks [22]. In one Australian study, discharge summaries were not available for $72 \%$ of the sample, especially for those aged 70 to 80 years, compared to those over 80 years old [23].

Finally, we examined factors affecting readmission rates and length of stay related to medication error and our findings highlight that medication-related hospitalisation and length of stay is a complex relationship and remains difficult to predict. Our study results do not reveal statistically significant associations between demographic, clinical, medication related factors and readmission rates and length of stay. However, the international literature reports on the prevalence and contributing factors to ADEs in developed and developing countries and reveals that adverse drug reactions were most common in older adults, female gender and with increased number of medications [24].

It is widely acknowledged that increasing interactions with and dependency on health care systems is common with advancing age [25]. In order for health care policy makers to optimise service utilisation for older adults who frequently transition between the hospital and home environments, evidence of inpatient hospitalisation patterns and precipitating circumstances in medication error are required [6]. In addition, the results of this study highlight the need for better continuity of care between hospital and primary healthcare services. A systematic review of approaches for improving continuity of care in medication management reported a need to move from epidemiological studies to well-designed intervention studies that include outcome measures and cost-effectiveness analysis [26].

\section{Strengths and Limitations}

The strengths of the study were that the research questions were well defined, inclusion and exclusion criteria were explicitly developed and the data abstractors were trained in reviewing charts and were monitored in the use of standar- 
dised, electronic abstraction forms. A particular strength was that medical clinicians were asked to determine whether medication error was a contributing factor to admission. Most other similar studies rely on admission diagnostic coding and a suspected contribution of a medication error may not be coded. Limitations relate to the study sample and design. Although the sample size was considered a priori, it used a small convenience sample due to time and resource constraints. The results of this study may be generalised to community-dwelling older adults in coastal/regional areas and caution should be used when generalising the results more broadly. The critical variables under examination were number of medications, regimen complexity and comorbidities. The scope of the research did not permit detailed examination of the types of medications contributing to medication error.

\section{Conclusions}

In this study of older community-dwelling persons in Australia, the prevalence of polypharmacy and medication regimen complexity at admission was high. A high proportion of older adults on medical units may have unrecognised medication errors impacting their admission. Medical discharge summaries inadequately addressed this issue for patients returning to the care of their GP. Consequently, predictive studies of the factors affecting readmission rates and length of stay related to medication error and inadequate discharge information need to be undertaken with larger sample sizes and involving multiple health care sites because the coastal communities of SEQ have a demographic that provides a view of the future demographic for Australia.

Currently, in Australia, the Domiciliary Medication Management Review (DMMR) may only be initiated by a patient's GP after assessing the patient's need for the service. In terms of policy change, there is an opportunity for nurses concerned about patient medication safety, in the transition to home, to advocate that the Discharge Summary include a request for the GP to assess medication administration safety and consider referral for a DMMR.

With regard to clinical practice imperatives, the results of this study confirm that there is a significant cohort of older persons who are discharged from the hospital not being confident and competent in the self- or carer-administration of medications. Hospital nurses are in an ideal position to assess patient and carer competence and, in collaboration with pharmacy staff, provide "just-in-time" medication education.

\section{Funding}

Financial assistance from the Collaborative Research grant supported the conduct of the study.

\section{Acknowledgements}

The research team wishes to thank our clinical investigators, Ms Sonya Wallace, 
Clinical Trials Nurse, for her role in data collection and data entry, and Ms Helen Rodgers, Nurse Unit Manager, Clinical Research, for database design and general study site management.

\section{Conflicts of Interest}

The authors declare no conflicts of interest regarding the publication of this paper.

\section{References}

[1] Wilson, R.M., Runciman, W.B., Gibberd, R.W., Harrison, B.T., Newby, L. and Hamilton, J.D. (1995) The Quality in Australian Health Care Study. The Medical Journal of Australia, 163, 458-471. https://doi.org/10.5694/j.1326-5377.1995.tb124691.x

[2] Mager, D. (2014) Hospitalization of Home Care Patients: Adverse Drug Events. Home Health Care Management and Practice, 26, 11-16. https://doi.org/10.1177/1084822313499772

[3] Graziano, O., van der Cammen, T.J.M., Petrovic, M., Somers, A. and Rajkumar, C. (2013) Strategies to Reduce the Risk of Iatrogenic Illness in Complex Older Adults. Age and Ageing, 42, 284-291. https://doi.org/10.1093/ageing/aft038

[4] Barnett, K., Mercer, S.W., Norbury, M., Watt, G., Wyke, S. and Guthrie, B. (2012) Epidemiology of Multimorbidity and Implications for Health Care, Research, and Medical Education: A Cross-Sectional Study. The Lancet, 380, 37-43. https://doi.org/10.1016/S0140-6736(12)60240-2

[5] Mangin, D., Heath, I. and Jamoulle, M. (2012) Beyond Diagnosis: Rising to the Multimorbidity Challenge. BMJ, 344, e3526. https://doi.org/10.1136/bmj.e3526

[6] Robinson, S., Howie-Esquivel, J. and Vlahov, D. (2012) Readmission Risk Factors after Hospital Discharge among the Elderly. Population Health Management, 5, 338-351. https://doi.org/10.1089/pop.2011.0095

[7] Salvi, F., Marchetti, A., D’Angelo, F., Boemi, M., Lattanzio, F. and Cherubini, A. (2012) Adverse Drug Events as a Cause of Hospitalization in Older Adults. Drug Safety, 35, 29-45. https://doi.org/10.1007/BF03319101

[8] Tangiisuran, B., Gozzoli, M.P., Davies, J.G. and Rajkumar, C. (2010) Adverse Drug Reactions in Older People. Reviews in Clinical Gerontology, 20, 246-259. https://doi.org/10.1017/S0959259810000171

[9] Garcia-Aparicio, J. and Herrero-Herrero, J.I. (2013) Medication Errors Detected in Elderly Patients Admitted to an Internal Medicine Service. International Journal of Clinical Practice, 67, 282-289. https://doi.org/10.1111/j.1742-1241.2012.02982.x

[10] Kalisch, L.M., Caughey, G.E., Barratt, J.D., Ramsay, E.N., Killer, G., Gilbert, A.L. and Roughead, E.E. (2012) Prevalence of Preventable Medication-Related Hospitalizations in Australia: An Opportunity to Reduce Harm. International Journal for Quality in Health Care, 24, 239-249. https://doi.org/10.1093/intqhc/mzs015

[11] Hain, D.J., Tappen, R., Diaz, S. and Ouslander, J.G. (2012) Cognitive Impairment and Medication Self-Management Errors in Older Adults Discharged Home from a Community Hospital. Home Healthcare Nurse, 30, 246-254. https://doi.org/10.1097/NHH.0b013e31824c28bd

[12] Stitt, D.M., Elliott, D.P. and Thompson, S.N. (2011) Medication Discrepancies Identified at Time of Hospital Discharge in a Geriatric Population. American Journal of Geriatric Pharmacotherapy, 9, 234-240.

https://doi.org/10.1016/j.amjopharm.2011.06.002 
[13] ABS (2012) Australian Demographic Statistics, March 2012/3101.0. Australian Bureau of Statistics, Canberra.

[14] RDASC. State of the Region Report 2012-2031. Regional Development Australia Sunshine Coast. Queensland. https://www.rdasunshinecoast.org.au

[15] Faul, F., Erdfelder, E., Buchner, A. and Lang, A.G. (2009) Statistical Power Analyses Using G*Power 3.1: Tests for Correlation and Regression Analyses. Behavior Research Methods, 41, 1149-1160. https://doi.org/10.3758/BRM.41.4.1149

[16] Charlson, M.E., Pompei, P., Ales, K.L. and MacKenzie, C.R. (1987) A New Method of Classifying Prognostic Comorbidity in Longitudinal Studies: Development and Validation. Journal of Chronic Diseases, 40, 373-383. https://doi.org/10.1016/0021-9681(87)90171-8

[17] Dias, A., Teixeira-Lopes, F., Miranda, A., Alves, M., Narciso, M., Mieiro, L., et al. (2015) Comorbidity Burden Assessment in Older People Admitted to a Portuguese University Hospital. Aging Clinical and Experimental Research, 27, 323-328. https://doi.org/10.1007/s40520-014-0280-5

[18] Beloosesky, Y., Weiss, A. and Mansur, N. (2011) Validity of the Medication-Based Disease Burden Index Compared with the Charlson Comorbidity Index and the Cumulative Illness Rating Scale for Geriatrics: A Cohort Study. Drugs Aging, 28, 1004-1014. https://doi.org/10.2165/11597040-000000000-00000

[19] George, J., Phun, Y.-T., Bailey, M.J., Kong, D.C.M. and Stewart, K. (2004) Development and Validation of the Medication Regimen Complexity Index. The Annals of Pharmacotherapy, 38, 1369-1376. https://doi.org/10.1345/aph.1D479

[20] Mudge, A.M., Shakhovoskoy, R. and Karrasch, A. (2013) Quality of Transitions in Older Medical Patients with Frequent Readmissions: Opportunities for Improvement. European Journal of Internal Medicine, 24, 779-783. https://doi.org/10.1016/j.ejim.2013.08.708

[21] Guthrie, B., Makubate, B., Hernandez-Santiago, V. and Dreischulte, T. (2015) The Rising Tide of Polypharmacy and Drug-Drug Interactions: Population Database Analysis 1995-2010. BMC Medicine, 13, Article No. 74. https://doi.org/10.1186/s12916-015-0322-7

[22] Bourgeois, F.T., Shannon, M.W., Valim, C. and Mandl, K.D. (2010) Adverse Drug Events in the Outpatient Setting: An 11-Year National Analysis. Pharmacoepidemiology and Drug Safety, 19, 901-910. https://doi.org/10.1002/pds.1984

[23] Grealish, L.A., Forbes, M., Beylacq, M., Adeleye, A.O. and Crilly, J. (2017) Older Persons Who Re-Present to the Emergency Department: An Observational Study. Collegian, 24, 557-562. https://doi.org/10.1016/j.colegn.2017.01.001

[24] Angamo, M.T., Chalmers, L., Curtain, C.M. and Bereznicki, L.R.E. (2016) Adverse-Drug-Reaction-Related Hospitalizations in Developed and Developing Countries: A Review of Prevalence and Contributing Factors. Drug Safety, 39, 847-857. http://rd.springer.com/journal/40264 https://doi.org/10.1007/s40264-016-0444-7

[25] Dierich, M.T., Mueller, C. and Westra, B. (2011) Medication Regimens in Older Home Care Patients. Journal of Gerontological Nursing, 37, 45-55. https://doi.org/10.3928/00989134-20111103-02

[26] Spinewine, A., Claeys, C., Foulon, V. and Chevalier, P. (2013) Approaches for Improving Continuity of Care in Medication Management: A Systematic Review. International Journal for Quality in Health Care, 25, 403-417. https://doi.org/10.1093/intqhc/mzt032 